\title{
Ultrawideband Impulse Radar Through-the-Wall Imaging with Compressive Sensing
}

\author{
Wenji Zhang, ${ }^{1,2}$ Moeness G. Amin, ${ }^{1}$ Fauzia Ahmad, ${ }^{1}$ Ahmad Hoorfar, ${ }^{1}$ \\ and Graeme E. Smith ${ }^{1,3}$ \\ ${ }^{1}$ Center for Advanced Communications, Villanova University, Villanova, PA 19085, USA \\ ${ }^{2}$ Department of Electrical Engineering, Duke University, Durham, NC, USA \\ ${ }^{3}$ The Ohio State University, Columbus, OH 43210, USA
}

Correspondence should be addressed to Wenji Zhang, wenjizhang@gmail.com

Received 6 December 2011; Accepted 13 February 2012

Academic Editor: Francesco Soldovieri

Copyright (c) 2012 Wenji Zhang et al. This is an open access article distributed under the Creative Commons Attribution License, which permits unrestricted use, distribution, and reproduction in any medium, provided the original work is properly cited.

\begin{abstract}
Compressive Sensing (CS) provides a new perspective for addressing radar applications requiring large amount of measurements and long data acquisition time; both issues are inherent in through-the-wall radar imaging (TWRI). Most CS techniques applied to TWRI consider stepped-frequency radar platforms. In this paper, the impulse radar two-dimensional (2D) TWRI problem is cast within the framework of CS and solved by the sparse constraint optimization performed on time-domain samples. Instead of the direct sampling of the time domain signal at the Nyquist rate, the Random Modulation Preintegration architecture is employed for the CS projection measurement, which significantly reduces the amount of measurement data for TWRI. Numerical results for point-like and spatially extended targets show that high-quality reliable TWRI based on the CS imaging approach can be achieved with a number of data points with an order of magnitude less than that required by conventional beamforming using the entire data volume.
\end{abstract}

\section{Introduction}

Through-the-wall radar imaging (TWRI) is a topic of current interest due to the wide range of public safety and defense applications. The TWRI technology is particularly useful in behind-the-wall target detection, surveillance and reconnaissance, law enforcement, and various earthquake and avalanche rescue missions [1-9].

Through-the-wall radars image the targets behind the wall by transmitting short pulses and processing the signal returns from the wall and targets. Several effective throughthe-wall beamformers that take into account the wall reflection, bending, and delay effects have been proposed for imaging of targets behind walls. Most of these algorithms are carried out in the frequency domain, such as the delay-andsum beamforming (DSBF) and the linear inverse scattering algorithms [3-9]. Both algorithms have an upper limit on the downrange and crossrange resolutions. The downrange resolution is restricted to $c / 2 B$, where $c$ is the speed of light and $B$ is the bandwidth of the signal, whereas the crossrange resolution is restricted to the diffraction tomography limit, which is related to the antenna array aperture size [10]. In order to achieve high resolution in both downrange and crossrange, an ultrawideband signal should be transmitted, and a long antenna array aperture should be synthesized. This results in a large amount of space-time/space-frequency data, long data acquisition time, and large storage and memory requirements. Reduction of the data volume is important in TWRI applications, as it accelerates processing and, subsequently, allows prompt actionable intelligence. It also relaxes constraints on system aperture and bandwidth and creates different design and deployment paradigms, which are more flexible than those underlying conventional SAR operations. It is also noted that data reduction and fast acquisition ease the requirement of slow target motion and/or stationarity, which could be necessary to avoid image smearing and imaged target displacements [11].

Recently, a new framework, referred to as compressive sensing (CS), for simultaneous sensing and compression has received considerable attention and has been successfully 
applied in many fields, that is, signal/image processing, radar imaging, communication, geophysics and remote sensing [12-23]. In CS, it has been shown that a signal/image, which is sparse or has a sparse representation in some basis, can be captured from a small number of random nonadapptive linear projections onto the measurement basis. The original signal can be reconstructed with incomplete measurement data through convex optimization that uses the sparsity as an important piece of a priori information [12-16]. The capability of CS to reconstruct a sparse signal from far fewer nonadapptive measurements provides a new perspective for data reduction in radar imaging without compromising the imaging quality. There have been several approaches that apply CS for radar imaging [18-24]. In [18, 21-23], CS is applied for synthetic aperture radar (SAR) imaging to obtain high-resolution imaging with very few measurements. Gurbuz et al. proposed a compressive sensing data acquisition and imaging method for step frequency continuous wave (SFCW) ground penetrating radar (GPR) [20], where the sparsity property and limited number of buried objects are successfully utilized for improved target detection and resolution. In [22, 24], similar data acquisition and target reconstruction strategies were applied for SFCW throughthe-wall radar imaging. The above-mentioned compressive GPR and TWRI algorithms are discussed in the framework of SFCW radar. As a viable alternative to SFCW radars, the impulse radar is commonly used for subsurface and through-the-wall imaging owing to its simple design and range resolution enhancement. The hardware design for impulse radar has been well-developed in the past few decades and it is now widely used in many applications. The signal measurement and processing for impulse radar are performed in the time domain, which enables easy employment of time-domain techniques. A data acquisition system was proposed in [19] for pulse GPR based on CS by exploiting the sparseness of point targets in the image space.

In this paper, we examine the application of CS to TWRI, which was introduced in [22] and further developed in [2428]. Specifically, we consider impulse radar platforms and deal with sampled radar returns in the time domain. In order to improve the imaging quality while significantly reducing the amount of data measurements and collection time, the image reconstruction problem is cast within the framework of CS and solved by the sparse constraint optimization. We assume that the scene is spatially sparse and no transform sparse representation is required [28]. By employing the point target model, a linear relation between the radar returns and the target space is obtained and represented in vector-matrix form. For the compressive TWRI system, a direct measurement of the received time-domain signal at the Nyquist rate is not applied. Instead, linear projections of the returned signals with random \pm 1 sequences, called random modulation preintegration (RMPI) architecture [13], are used to reduce the number of CS measurements below the conventional Nyquist sampling rate, without noticeable degradation of the image quality. With the informative CS measurement data, the TWRI is solved as sparse constraint optimization problem which well-exploits the sparsity of the targets space and enables high-resolution, less-cluttered

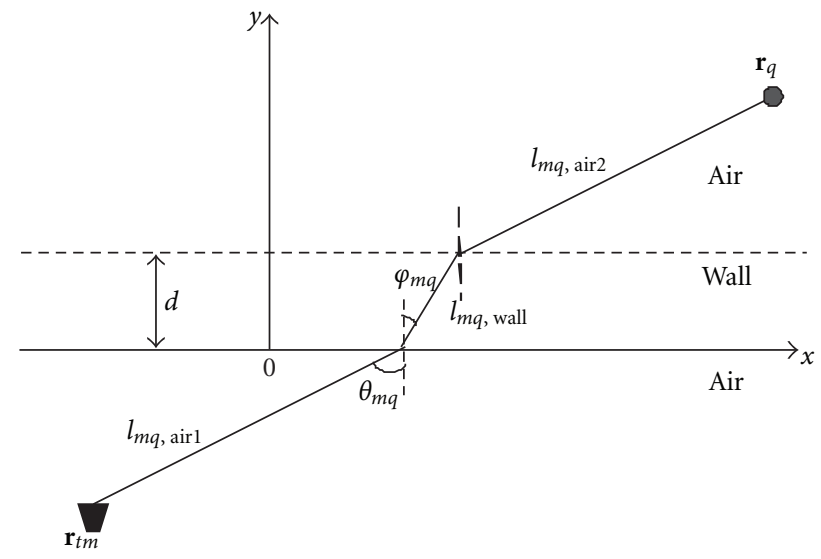

FIGURE 1: TWRI geometry.

images. We closely follow the development in [19], but apply CS to TWRI, rather than GPR, using EM modeling examples of point and spatially extended targets. The latter includes a human. It is important to note that in this paper, we assume that wall mitigation techniques have already been applied to remove the strong wall backscattering, allowing the targets to be electromagnetically visible to the imaging system. Recent techniques combining CS and wall mitigation for steppedfrequency radars were discussed in $[29,30]$.

The organization of the remainder of the paper is as follows. In Section 2, we first describe the TWRI signal model and then provide details of the compressive sensing data acquisition, and the impulse radar CS imaging algorithm for TWRI. We present simulation results and discussions in Section 3. Finally, conclusions are drawn in Section 4.

\section{Compressive Sensing for Impulse Radar Through-the-Wall Imaging}

2.1. Signal Model. Impulse through-the-wall radar scans a region of interest by transmitting short pulses and processing the reflected signals. Figure 1 depicts a simple TWRI scenario using monostatic SAR. The transceiver interrogates the targets behind the wall with a short pulse $s(t)$ at $M$ locations. The coordinates of the $m$ th transceiver location are denoted as $\mathbf{r}_{t m}=\left(x_{t m}, y_{t m}\right)$. The targets are located in an inaccessible investigated region behind the wall. The permittivity and thickness of the wall are denoted as $\varepsilon_{r}$ and $d$, respectively.

For a single-point target located at $\mathbf{r}_{q}=\left(x_{q}, y_{q}\right)$, by employing the point target model, the received signal at the $m$ th receiver can be written as

$$
\beta_{m}(t)=\alpha_{q} s\left(t-\tau_{m}(q)\right)
$$

where $\alpha_{q}$ is the reflectivity of the point target and $\tau_{m}(q)$ is the propagation delay from the $m$ th transmitter to the $q$ th target. The point target model is based on first order Born approximation, which ignores the multiple scattering effect. Thus, the received signal is essentially a delayed and scaled version of the transmitted pulse. 
Given the exact knowledge of the wall permittivity and thickness, the propagation delay $\tau_{m}(q)$ from the $m$ th transceiver to the qth target can be calculated as [31]

$$
\tau_{m}(q)=2\left(\frac{l_{m q, \text { air } 1}+l_{m q, \text { air } 2}}{c}+\frac{l_{m q, \text { wall }}}{v}\right) .
$$

As depicted in Figure 1, $l_{m q \text {,air }}$ is the wave traveling distance from the $m$ th transmitter to the wall, $l_{m q \text {,air } 2}$ is the wave traveling distance beyond the wall to the target for the $m$ th transceiver, $l_{m q \text {,wall }}$ is the wave traveling distance inside the wall, and $c$ and $v$ are the wave propagation speeds in the air and the wall, respectively.

The analytical expressions for the calculation of the distances $l_{m q, \text { air } 1}, l_{m q, \text { air } 2}$, and $l_{m q \text {,wall were derived in }[3,7,31]}$ and are given by

$$
l_{m q, \text { airl }}=\frac{y_{t m}}{\cos \theta_{m q}}, \quad l_{m q, \text { air } 2}=\frac{y_{q}-d}{\cos \theta_{m q}}, \quad l_{m q, \text { wall }}=\frac{d}{\cos \varphi_{m q}},
$$

where $\theta_{m q}$ is the angle of incidence and $\varphi_{m q}$ is the angle of refraction for the $m$ th transceiver, which can be computed by solving the following equation $[3,7]$ :

$$
\begin{aligned}
\left(x_{q}-\right. & \left.\left(x_{t m}+y_{t m} \tan \theta_{m q}\right)\right)^{2}+y_{q}^{2} \\
= & l_{m q, \text { wall }}^{2}+l_{m q, \text { air } 2}^{2} \\
& \quad-2 l_{m q, \text { wall }} l_{m q, \text { air } 2} \cos \left(\pi+\varphi_{m q}-\theta_{m q}\right) .
\end{aligned}
$$

The incident and refraction angles satisfy the Snell's law

$$
\varphi_{m q}=\sin ^{-1}\left(\frac{\left(\sin \theta_{m q}\right)}{\sqrt{\varepsilon_{r}}}\right) \text {. }
$$

Suppose $Q$ point targets are located behind the wall. The received signal at the $m$ th receiver is then a superposition of echoes of the transmitted pulse:

$$
\beta_{m}(t)=\sum_{q=1}^{Q} \alpha_{q} s\left(t-\tau_{m}(q)\right)
$$

In the above formulation, we have assumed that the returns from the wall itself have been removed or mitigated and we only consider the effect of the wall on signal transmission as it propagates to and from the target. Wall return mitigation has been addressed in several recent publications $[6,32-34]$. In $[6,34]$, the wall thickness and dielectric constant are estimated and used to model the wall returns, which are then subtracted from the measurement data. The approaches in $[32,33]$, on the other hand, do not estimate the wall parameters, but they rather remove wall reflections by relying on the similarity and the strength of the wall returns measured from different antenna positions parallel to the wall. Spatial filtering and subspace projection methods can be applied for this purpose.
2.2. Compressive Impulse Through-the-Wall Radar Data Acquisition. Conventional time-domain imaging uses the standard backprojection method to reconstruct the target scene $[5,19]$. In order to achieve a high crossrange resolution, the radar needs to synthesize a long array aperture with a fine spatial sampling rate. Meanwhile, in order to perform matched filtering of the received data, the time-domain data should be sampled at least at the Nyquist rate. This results in a large amount of data from collection time perspective and still cannot break the conventional resolution limits. On the other hand, in CS, $O(K \log (N))$ measurements are sufficient for reconstructing exactly a $\mathrm{K}$-sparse $\mathrm{N}$-dimensional signal via $L_{1}$ minimization, regardless of the positions of the $K$ nonzero elements, which can be either adjacent or separated. This implies that not only reconstruction of the sparse target can be achieved with a limited number of incoherent measurements, but also we can resolve two closely spaced targets that are otherwise unresolved by the point spread function associated with conventional imaging.

Sparsity of the target space is an important a priori piece of information, which has only been recently exploited in step-frequency TWRI $[22,25-28]$. In TWRI, the region of interest is divided into a finite number of pixels in crossrange and downrange. The sparsity in TWRI implies that the number of target pixels is much smaller than the total number of pixels in the image, which holds for most TWRI applications.

For $Q$ pixels constituting the target space, that is, the region being imaged, the image pixel values can be related to the received data in the matrix form using (6) as follows:

$$
\boldsymbol{\beta}_{m}=\boldsymbol{\Psi}_{m} \boldsymbol{\alpha}
$$

In (7), $\boldsymbol{\beta}_{m}=\left[\beta_{m}\left(t_{0}\right), \beta_{m}\left(t_{1}\right), \ldots, \beta_{m}\left(t_{N_{t}-1}\right)\right]^{T}$, where $t_{0}$ is the initial measurement time, $t_{N_{t}-1}=t_{0}+\left(N_{t}-1\right) \Delta t, \Delta t$ is the sampling time, which should be higher than the Nyquist sampling rate, and $N_{t}$ is the total number of time samples. The $q$ th column of the $N_{t} \times Q$ matrix $\Psi_{m}$ is given by [19]

$$
\left[\Psi_{m}\right]_{q}=\frac{s\left(t-\tau_{m}(q)\right)}{\left\|s\left(t-\tau_{m}(q)\right)\right\|_{2}}, \quad t=t_{0}, \ldots, t_{N_{t}-1} .
$$

Further, $\boldsymbol{\alpha}=\left[\alpha_{1}, \alpha_{2}, \ldots, \alpha_{Q}\right]^{T}$ in (7) is a vector indicating the property of the target space. That is, if there is a target at the $q$ th pixel, the value of the $q$ th element of $\boldsymbol{\alpha}$ should be nonzero; otherwise, $\alpha_{q}=0$.

For compressive impulse TWRI, instead of directly measuring the $N_{t}$-dimensional signal $\boldsymbol{\beta}_{m}$, we measure its projection on a basis $\boldsymbol{\Phi}_{m}$

$$
\mathbf{z}_{m}=\boldsymbol{\Phi}_{m} \boldsymbol{\beta}_{m}=\boldsymbol{\Phi}_{m} \boldsymbol{\Psi}_{m} \boldsymbol{\alpha},
$$

where $\Phi_{m}$ is a $J \times N_{t}$ measurement matrix and $J<<$ $N_{t}$. In order to achieve the minimum coherence of the measurement matrix with the matrix $\Psi_{m}$, some typical random matrices are proven to be universal, such as the Gaussian random matrix and the Rademacher matrix [1215]. The Gaussian random matrix is constructed by sampling independent, identically distributed entries from the Gaussian distribution $N(0,1)$. The Rademacher matrix has 


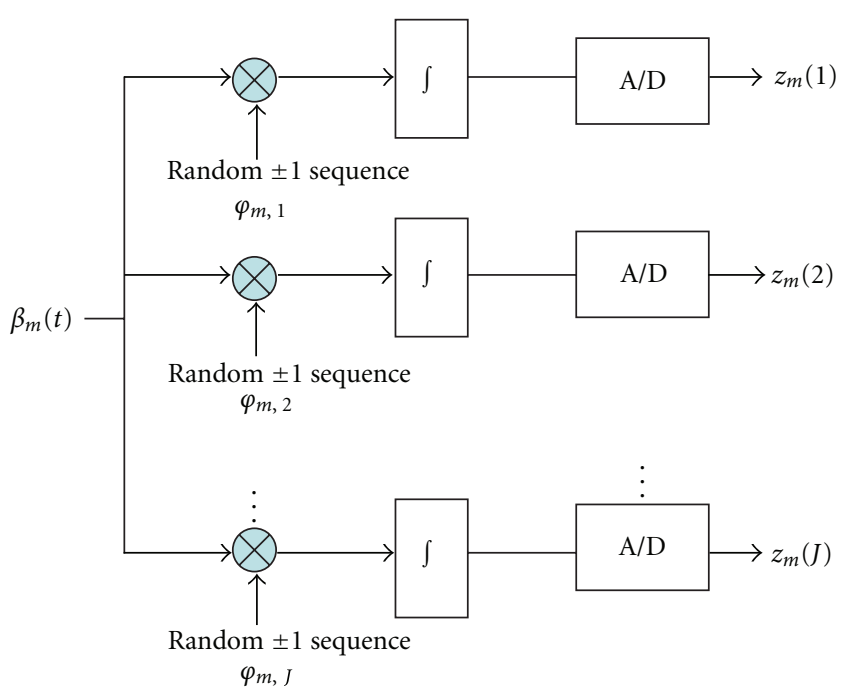

FIGURE 2: RMPI architecture for the hardware implementation of compressive impulse through-the-wall radar.

random \pm 1 entries with probability of $1 / 2$, which satisfies the symmetric Bernoulli distribution. Considering the hardware complexity and cost, it is much simpler to generate a random \pm 1 sequence than the random Gaussian sequence. In this paper, the RMPI architecture in [13] is adopted for the implementation of the impulse TWRI radar CS projection measurements (see Figure 2). In the RMPI architecture, the random sequences can be efficiently generated using field programmable gate array (FPGA). The generated sequences are used in the optimization process. By employing the above data acquisition strategy, the random measurement matrix condenses the $N_{t}$-dimensional signal $\boldsymbol{\beta}_{m}$ into a $J\left(<<N_{t}\right)$ dimensional signal $\mathbf{z}_{m}$.

2.3. Compressive Impulse TWRI. As depicted in Figure 1, the transceiver interrogates the targets behind the wall with a short pulse along a scan line at $M$ locations. For coherent processing of the received data, we can superpose the matrix equation in (9) for all $M$ illumination locations to form a large matrix:

$$
\mathrm{z}=\Phi \Psi \alpha=\Theta \alpha
$$

where

$$
\begin{gathered}
\mathbf{z}=\left[\mathbf{z}_{1}^{T}, \mathbf{z}_{2}^{T}, \ldots, \mathbf{z}_{M}^{T}\right]^{T}, \quad \boldsymbol{\Phi}=\operatorname{diag}\left[\boldsymbol{\Phi}_{1}, \boldsymbol{\Phi}_{2}, \ldots, \boldsymbol{\Phi}_{M}\right], \\
\boldsymbol{\Psi}=\left[\boldsymbol{\Psi}_{1}^{T}, \boldsymbol{\Psi}_{2}^{T}, \ldots, \boldsymbol{\Psi}_{M}^{T}\right]^{T}, \quad \boldsymbol{\Theta}=\boldsymbol{\Phi} \boldsymbol{\Psi} .
\end{gathered}
$$

The target space can be reconstructed by solving the following basis pursuit problem $[13,35]$ :

$$
\min _{\boldsymbol{\alpha}}\|\boldsymbol{\alpha}\|_{1} \quad \text { subject to } \mathbf{z}=\boldsymbol{\Theta} \boldsymbol{\alpha} \text {. }
$$

For radar imaging applications, the measurement data is always contaminated with noise. For noisy measurements, (10) can be written as

$$
\mathbf{z}=\boldsymbol{\Theta} \alpha+\mathbf{u}
$$

where $\mathbf{u}=\left[\mathbf{u}_{1}^{T}, \mathbf{u}_{2}^{T}, \ldots, \mathbf{u}_{M}^{T}\right]^{T}$ and $\mathbf{u}_{m}$ is the measurement noise at the $m$ th antenna location. From [20, 24], robust reconstruction of a sparse signal under noise corrupted data can be achieved by solving the following convex optimization problem, which is also referred to as Dantzig selector:

$$
\min _{\boldsymbol{\alpha}}\|\boldsymbol{\alpha}\|_{1} \text { subject to }\left\|\Theta^{T}(\mathbf{z}-\Theta \boldsymbol{\alpha})\right\|_{\infty}<\delta,
$$

where $\delta$ represents a small tolerance error. All matrices and vectors in this paper are real valued, thus (14) is a Linear Programming (LP) problem and can be efficiently solved using the interior point method [35]. In this paper, the Dantzig selector solver in the sparse constraint optimization package $L_{1}$-magic [36] is employed for solving (14). In the optimization procedure, the choice of the error $\delta$ is the key to rendering desirable results. An improper choice of the error will probably lead to missing true targets, introducing false targets, or even cause divergence with increased iterations. We employ the cross-validation (CV) strategy for an automatic selection of the error value in the optimization process. In essence, the cross-validation strategy divides the measurements data into estimation and cross validation sets. The steps of the CV-based optimization can be described as follows [20,37]:

(1) Initialization. Set $\delta=\gamma\left\|\Theta_{E}^{T} \mathbf{z}_{E}\right\|_{\infty}, i=1$, where $\gamma<1$.

(2) Estimation. Solve (14) to estimate the target location $\boldsymbol{\alpha}^{(i)}$ with the estimation data set $\mathbf{z}_{E}, \boldsymbol{\Theta}_{E}$.

(3) Cross-validation. If $\left\|\boldsymbol{\Theta}_{\mathrm{CV}}^{T}\left(\mathbf{z}_{\mathrm{CV}}-\boldsymbol{\Theta}_{\mathrm{CV}} \boldsymbol{\alpha}^{(i)}\right)\right\|_{\infty}<\delta$, set $\delta=$ $\left\|\boldsymbol{\Theta}_{\mathrm{CV}}^{T}\left(\mathbf{z}_{\mathrm{CV}}-\boldsymbol{\Theta}_{\mathrm{CV}} \boldsymbol{\alpha}^{(i)}\right)\right\|_{\infty}$, else terminate the iteration.

(4) Iteration. Increase $i$ by 1 and go to Step (2). where the subscripts $E$ and $C V$ stand for the estimation and cross validation data sets, respectively.

\section{Results and Discussions}

In order to demonstrate the effectiveness of the proposed approach for ultrawideband impulse TWRI with compressive sensing, several numerical examples are presented in this section.

The first simulation is the reconstruction of three point targets which are located at $(-0.15 \mathrm{~m}, 0.4 \mathrm{~m}),(0,0.6 \mathrm{~m})$, and $(0.1 \mathrm{~m}, 0.5 \mathrm{~m})$ in the $(x, y)$ plane. The transceiver array illuminates the targets at a distance of $0.3 \mathrm{~m}$ from the wall, along a line from $-1 \mathrm{~m}$ to $1 \mathrm{~m}$ with a step of $0.05 \mathrm{~m}$. The dielectric constant and thickness of the wall are $\varepsilon_{r}=6$ and $d=0.2 \mathrm{~m}$, respectively. The excitation signal is a modulated Gaussian pulse which covers the frequency range from $0.6 \mathrm{GHz}$ to $3.2 \mathrm{GHz}$ (as shown in Figure 3(a)). The time step used in the simulation is $\Delta t=19.245 \mathrm{ps}$ and the total number of time steps is $N_{t}=600$.

The conventional pulse radar collects the time-domain response from the targets at each receiving location and the measurement data is shown in Figure 3(b), which consists of $41 \times 600$ space-time measurements. Instead of the direct 


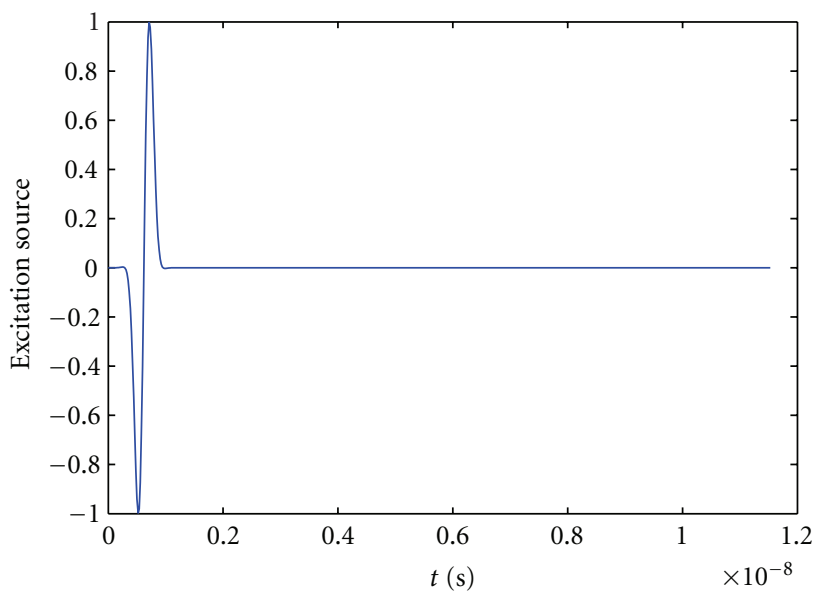

(a)

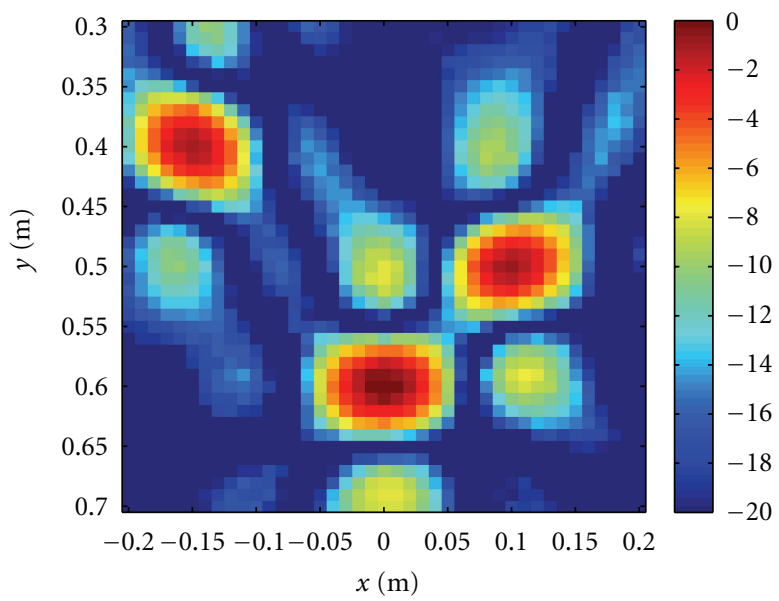

(c)

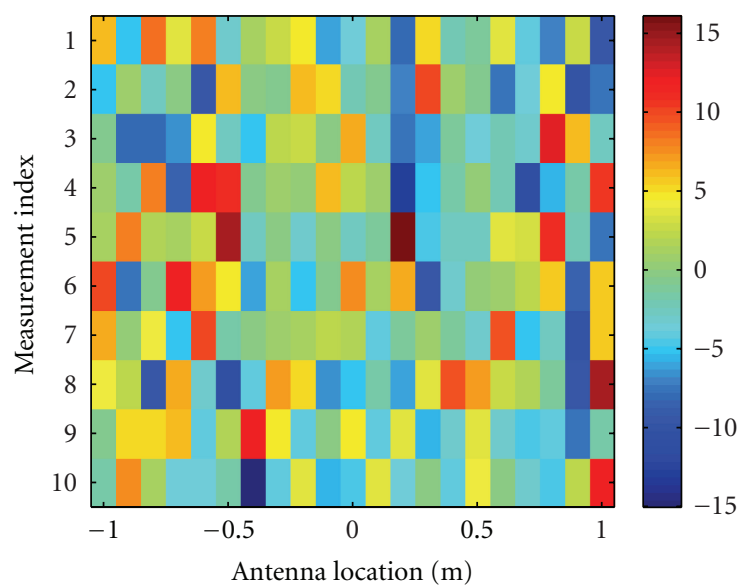

(e)

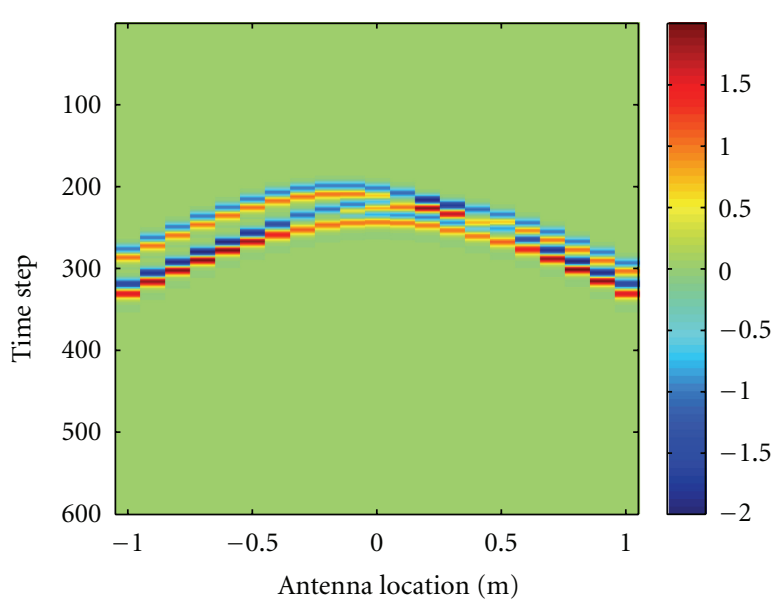

(b)

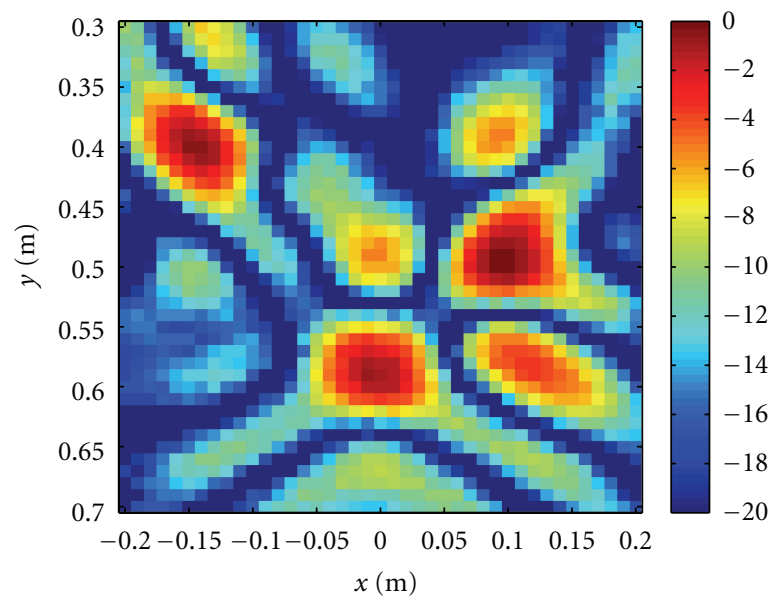

(d)

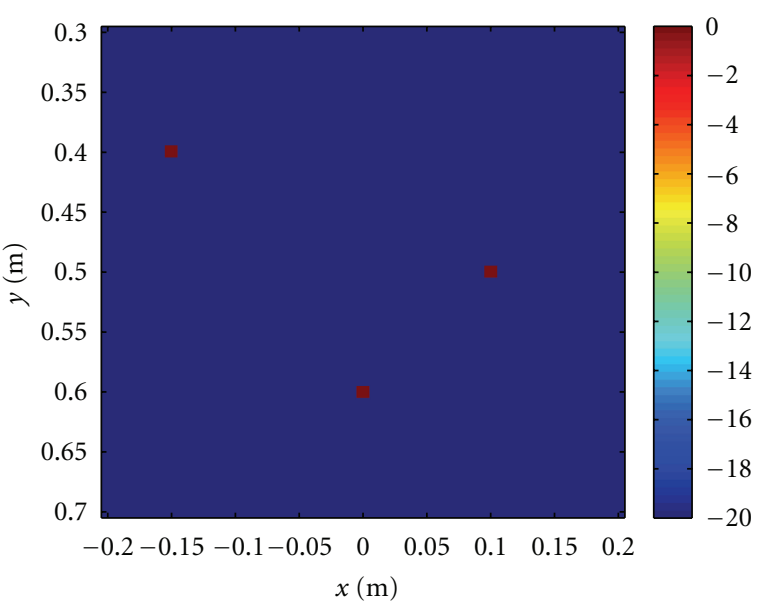

(f)

FIGURE 3: Imaging results of three point targets: (a) Excitation pulse, (b) Conventional radar measurement, (c) TDBP result with full data, (d) TDBP result with undersampled data, (e) CS measurement, and (f) CS result. 


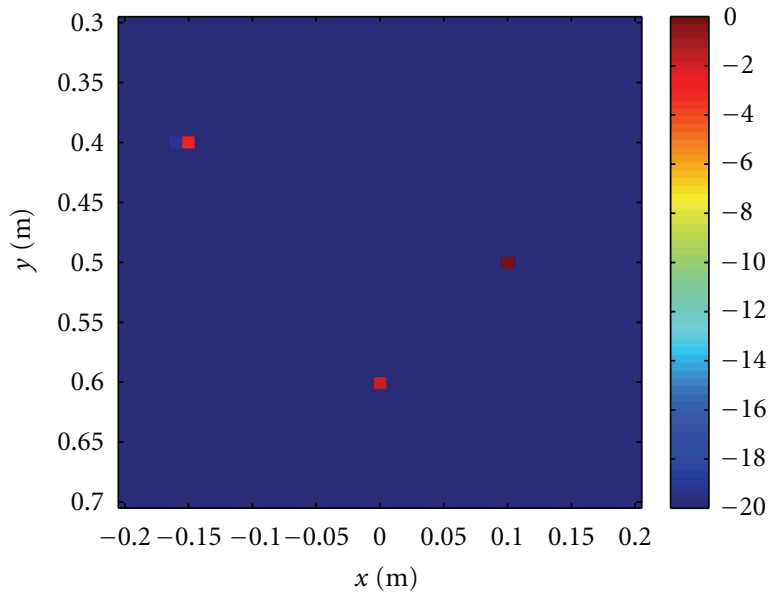

(a)

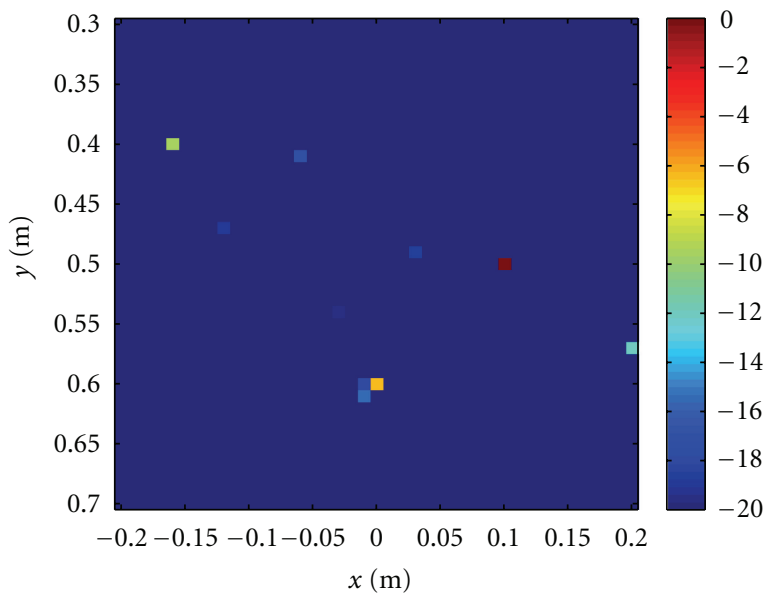

(b)

FIGURE 4: CS imaging results under different noise levels (a) SNR $=5 \mathrm{~dB}$; (b) $\mathrm{SNR}=-5 \mathrm{~dB}$.

measurement of the time-domain signal, CS makes 10 inner product measurements at each antenna location. This was carried out in the simulation by taking the product of the time-domain raw data with rows of a random Rademacher matrix of size $10 \times 600$. In the simulation, the size of the image to be constructed is $0.4 \mathrm{~m} \times 0.4 \mathrm{~m}$, which is divided into $40 \times 40$ pixels, resulting in 1600 unknowns for the sparse constraint optimization. Figure $3(\mathrm{c})$ is the time-domain backprojection (TDBP) imaging result using the $41 \times 600$ space-time measurements of Figure 3(b). If only 10 uniform samples are taken from Figure 3(b) at each antenna location, the TDBP imaging result is blurred, distorted, and has higher sidelobe levels, as evident from Figure 3(d). Figure 3(f) depicts the imaging result by solving the sparse constraint optimization problem of (14) with 10 projection measurements (shown in Figure 3(e)) at each antenna location. From the CS reconstructed image, the three targets are clearly identified at the true positions. That is, using the same amount of data as in Figure 3(d), a much higher resolution and cleaner image can be obtained through sparse constraint optimization with the CS projection measurements. The compressive radar imaging system condenses the large amount of data into far fewer incoherent projection measurements, which carry independent information of the targets.

The simulation results in Figure 3 are performed for the noiseless case. In order to demonstrate the performance of the proposed approach for noisy data, we compute the imaging results for the three point targets scene, contaminated by additive Gaussian random noise with signal to noise ratio (SNR) from $-15 \mathrm{~dB}$ to $20 \mathrm{~dB}$; only the results for $\mathrm{SNR}= \pm 5 \mathrm{~dB}$ are shown in Figure 4 . We observe that the CS technique suffers under low signal-to-noise ratio conditions and some false targets appear in the reconstructed image as a result of the reconstruction residual, which basically increases with the noise level. This phenomenon has been previously reported in $[20,22,24]$. We characterize the imaging quality under different noise levels by the mean

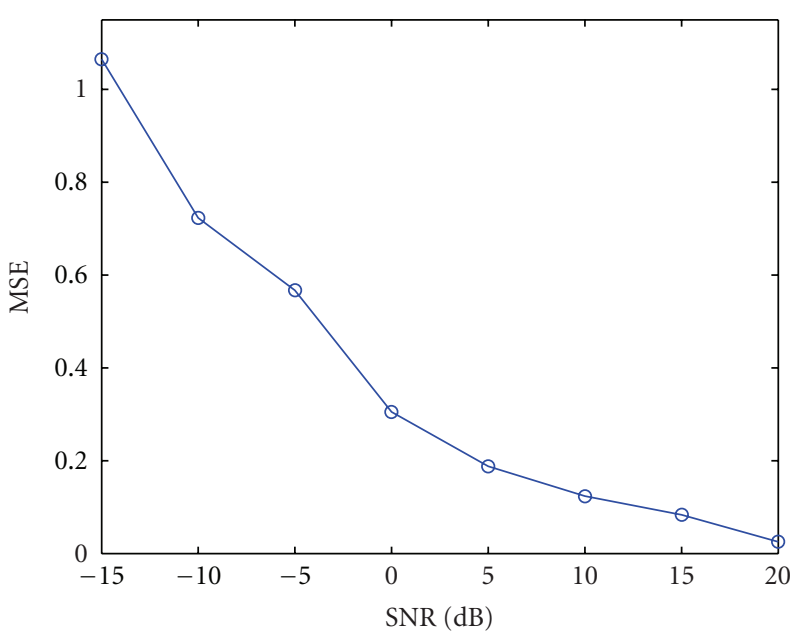

Figure 5: MSE under different noise levels.

squared error (MSE), which is plotted in Figure 5. The MSE is defined as the norm of the error between the reconstructed image and the ground truth image, normalized to the norm of the ground truth image. From Figure 5, we find that the MSE is reduced with increasing SNR.

It has been shown that the imaging resolution beyond that of conventional pulse radar can be obtained by using sparse constraint optimization $[20,25]$. In order to demonstrate superresolution in TWRI using the proposed approach, the CS imaging result of three closely spaced point targets is presented in Figure 6(a). The working conditions are the same as the previous simulation example, except that the three point targets are now located at $-0.05 \mathrm{~m}, 0.5 \mathrm{~m}$, $-0.05 \mathrm{~m}, 0.45 \mathrm{~m}$, and $0,0.5 \mathrm{~m}$. For comparison, the TDBP imaging result using the full set of $41 \times 600$ space-time data points is provided in Figure 6(b). From Figure 6, we observe that, even with the full space-time data, we are unable to 


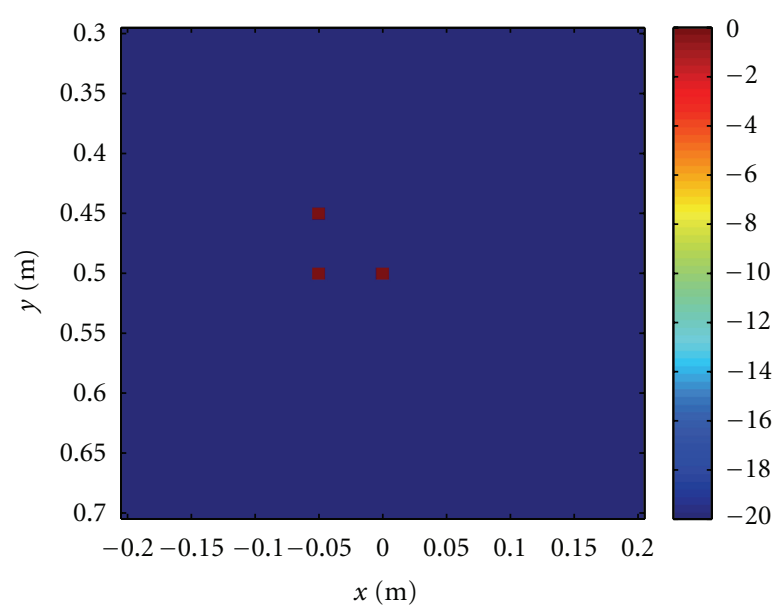

(a)

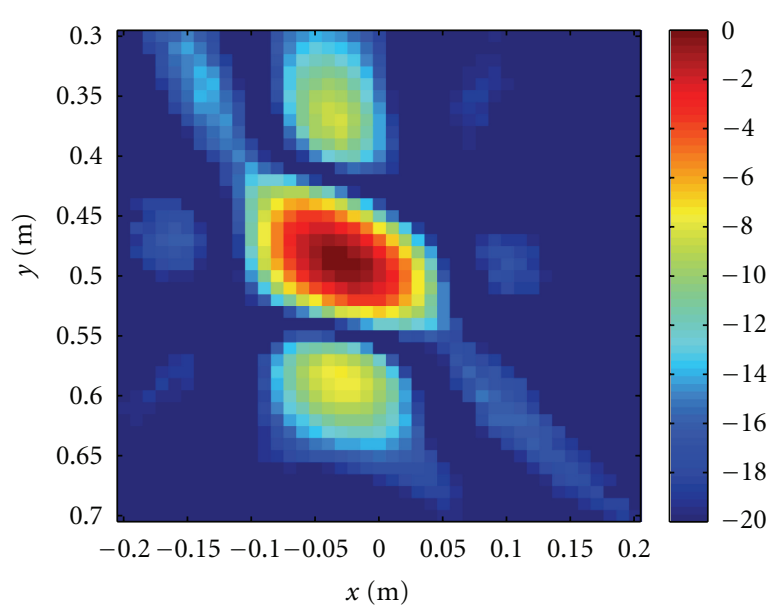

(b)

FIGURE 6: CS and TDBP imaging results of three closely spaced targets: (a) CS result and (b) TDBP result with full data.

TABLE 1: Target spatial characteristics.

\begin{tabular}{lccc}
\hline & Target type & Size & Center position \\
\hline Target 1 & Square & $0.2 \mathrm{~m} \times 0.2 \mathrm{~m}$ & $(-0.5 \mathrm{~m}, 1.1 \mathrm{~m})$ \\
Target 2 & Cylindrical & Radius $=0.1 \mathrm{~m}$ & $(0,1.0 \mathrm{~m})$ \\
Target 3 & Rectangular & $0.3 \mathrm{~m} \times 0.2 \mathrm{~m}$ & $(0.65 \mathrm{~m}, 1.2 \mathrm{~m})$ \\
\hline
\end{tabular}

resolve the three targets using the conventional TDBP algorithm. However, by using the CS projection measurement and through sparse constraint optimization, the three point targets are clearly resolved, and superresolution imaging is achieved.

The effectiveness of the proposed TWRI approach for spatially extended targets is shown by the following two simulations examples. In the first example, three twodimensional (2D) perfectly electrically conducting (PEC) objects (as shown in Figure 7(a)) are considered. The spatial characteristics of the three targets are specified in Table 1. The measurement data is generated using 2D FiniteDifference Time-Domain (FDTD) code. The monostatic radar measures the return signal at a distance of $0.3 \mathrm{~m}$ from the wall over a synthetic aperture extending from $-1 \mathrm{~m}$ to $1 \mathrm{~m}$ with a step of $0.05 \mathrm{~m}$. The dielectric constant and thickness of the wall are $\varepsilon_{r}=7.66$ and $d=0.2 \mathrm{~m}$, respectively. The time step is $\Delta t=11.79 \mathrm{ps}$, and the total number of time steps is $N_{t}=2120$. The investigation region is $2 \mathrm{~m} \times 1.6 \mathrm{~m}$ and divided into $49 \times 40$ pixels. The space-time response signal from the aforementioned targets is shown in Figure 7(b), which consists of $41 \times 2120$ time-domain raw data. Figure 7(c) is the TDBP imaging result using the full measured data of Figure 7(b). For CS, we only take 20 projection measurements at each of the 41 antenna locations, which results in a total of 820 CS measurements, shown in Figure 7(d). Figure 7(e) is the CS imaging result by solving the sparse constraint optimization problem in (14). One-sixth of the 820 data points were used for the cross validation, while the remaining were used for the estimation. From Figure 7(e), we observe that, even with only 20 measurements taken at each antenna location, the three targets are clearly observed at their true locations. In order to give a qualitative description of the imaging result, the image SNR (ISNR) defined in [38] is introduced for the characterization of the imaging quality. The ISNR is defined as follows:

$$
\operatorname{ISNR}=10 \log \left\{\frac{Q-Q_{R}}{Q_{R}} \times \frac{\sum_{i=1}^{Q_{R}}\left|\mu_{i}\right|^{2}}{\sum_{j=1}^{Q-Q_{R}}\left|\eta_{j}\right|^{2}}\right\},
$$

where $Q$ is the total number of pixels in the normalized image, with $Q_{R}$ pixels whose intensity is within -10 to $0 \mathrm{~dB},\left|\mu_{i}\right|^{2}$ is the image pixel intensity value above $-10 \mathrm{~dB}$, and $\left|\eta_{j}\right|^{2}$ is the image pixel intensity of the remaining $Q-Q_{R}$ pixels. For the CS imaging result of Figure 7(e), the ISNR is $65.8 \mathrm{~dB}$ whereas the ISNR for the TDBP imaging result of Figure $7(\mathrm{c})$ is $17.3 \mathrm{~dB}$. Along with a significant reduction in the amount of data, the proposed CS imaging approach provides superior performance over TDBP in terms of the ISNR.

To illustrate the performance of the CS scheme under noisy measurements from a scene of spatially extended targets, we provide in Figure 7(e) an image of the scene in Figure 7(a) corresponding to an SNR of $0 \mathrm{~dB}$. Similar to the CS results for imaging of point targets under noisy conditions, the scene reconstruction in Figure 7(e) successfully localized the three targets, but does suffer from several false alarms.

Finally, the simulation results for a human behind a homogeneous concrete wall are presented. The measurement configuration is shown in Figure 8(a), along with the front and side view of the human. The dimension of the high fidelity (HiFi) male human model is $0.57 \mathrm{~m} \times 0.324 \mathrm{~m} \times$ $1.88 \mathrm{~m}$ and is made up of $2.9 \mathrm{~mm}$ cubical FDTD mesh cells, consisting of 23 different tissue types. The radar standoff distance from the wall and the synthetic aperture array are the same as in the previous FDTD example. 


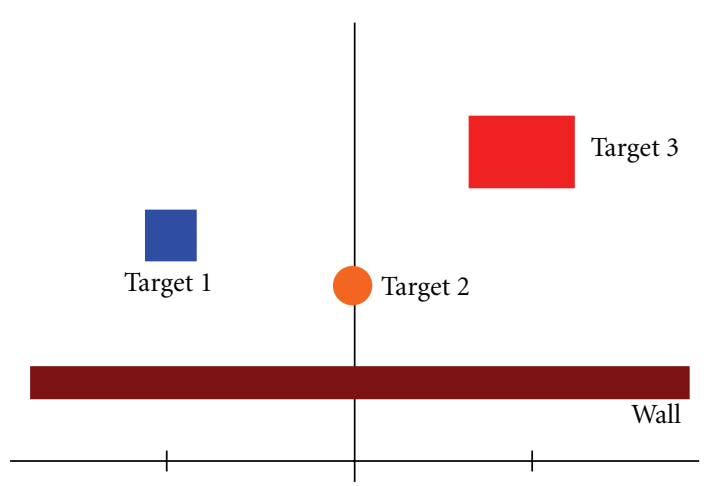

(a)

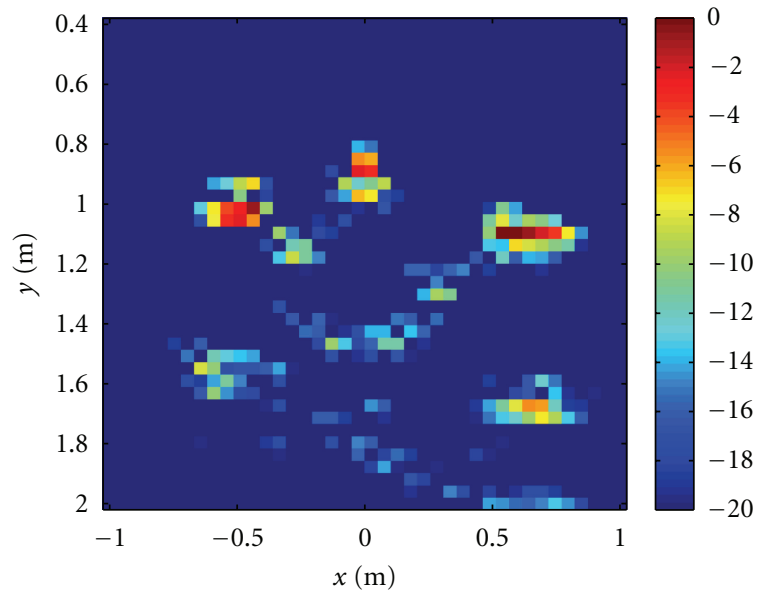

(c)

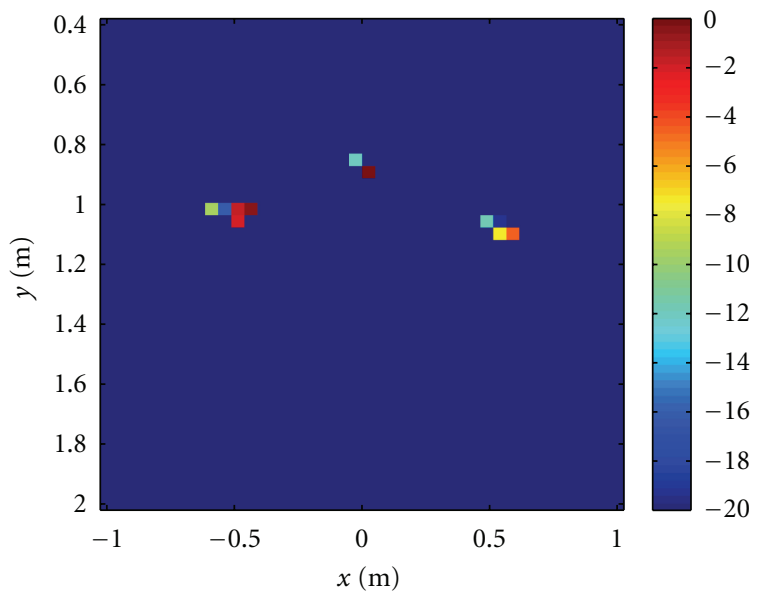

(e)

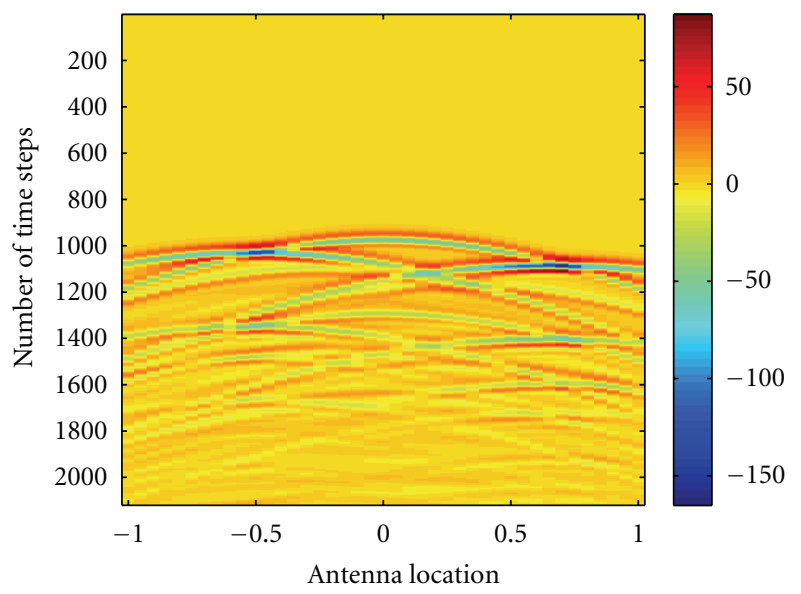

(b)

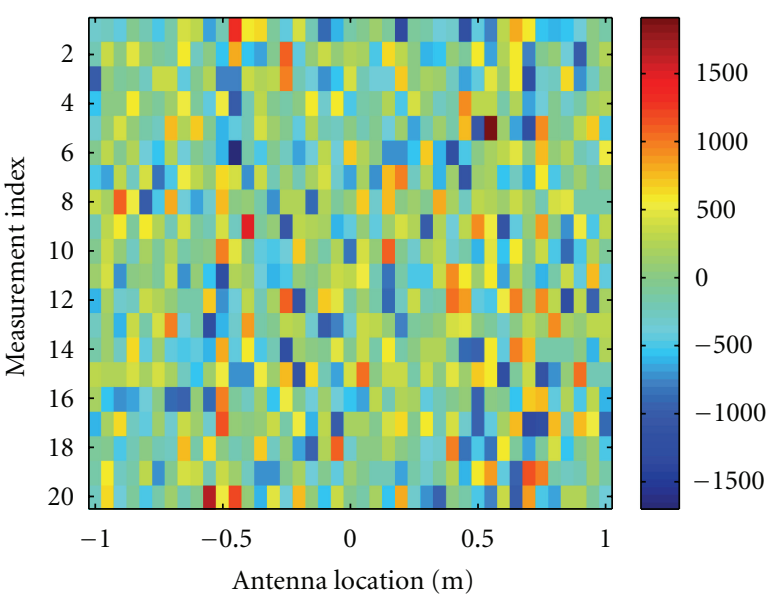

(d)

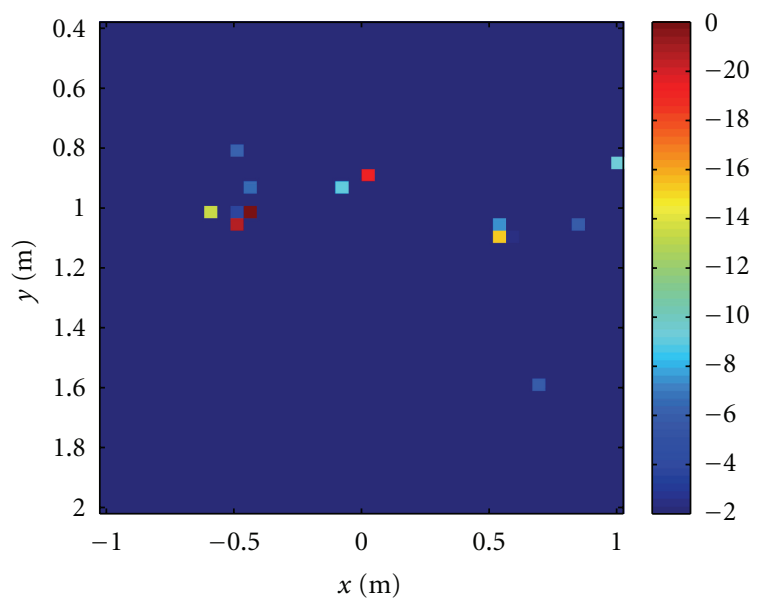

(f)

FIGURe 7: Imaging results of three PEC objects: (a) Target scene, (b) Conventional pulse radar measurement, (c) TDBP result, (d) CS measurement, (e) CS result, and (f) CS result under SNR $=0 \mathrm{~dB}$.

The REMCOM XFDTD numerical simulator was used for generation of the measurement data. The dielectric constant and thickness of the wall are $\varepsilon_{r}=6$ and $d=0.2 \mathrm{~m}$, respectively. The time step is $\Delta t=11.55 \mathrm{ps}$ and the total number of time steps is $N_{t}=2400$. The received time domain signal at each antenna location is shown in Figure $8(\mathrm{~b})$. Figure $8(\mathrm{c})$ is the TDBP imaging result using the full measured data of Figure 8(b). For the CS measurement, 

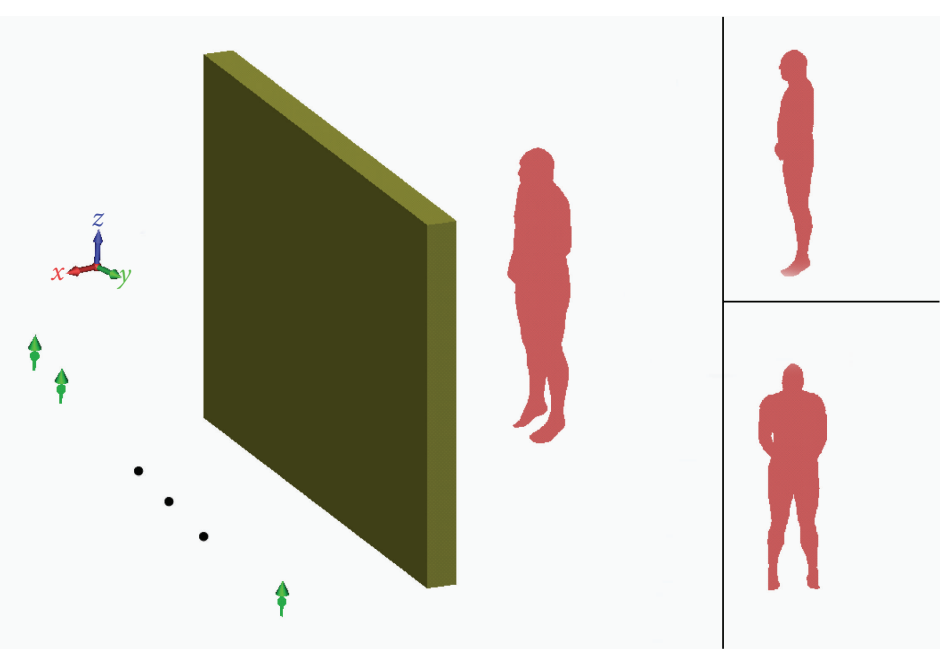

(a)

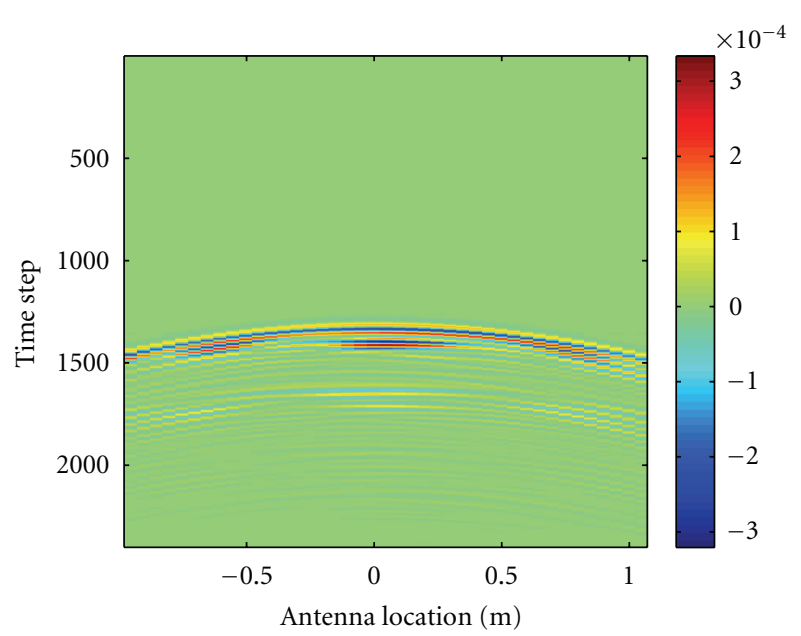

(b)

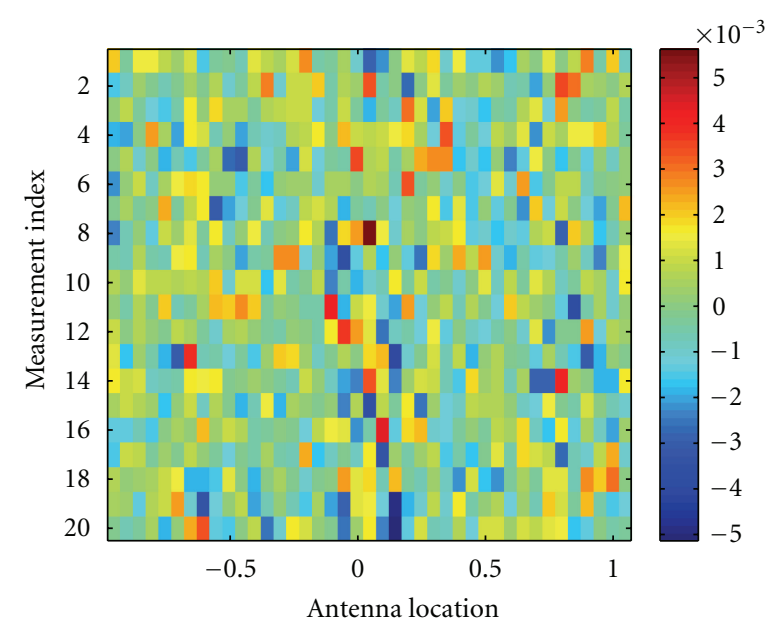

(d)

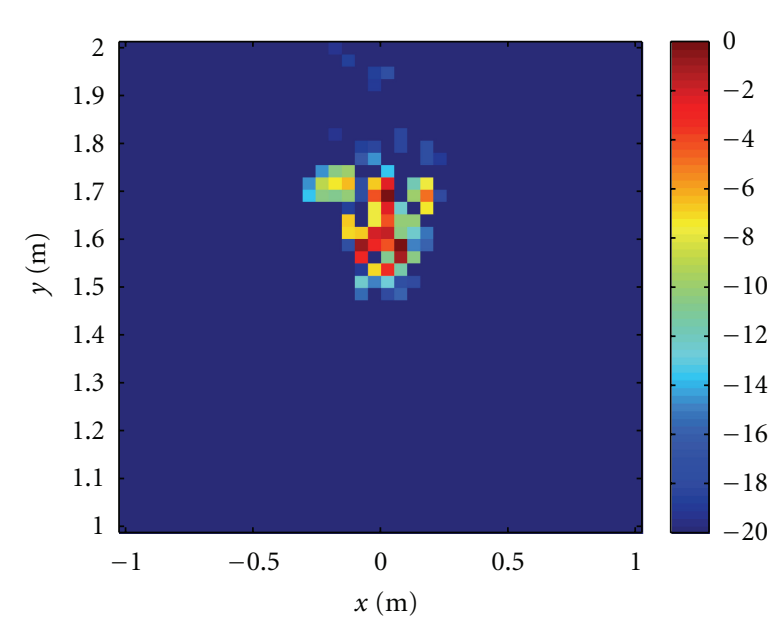

(c)

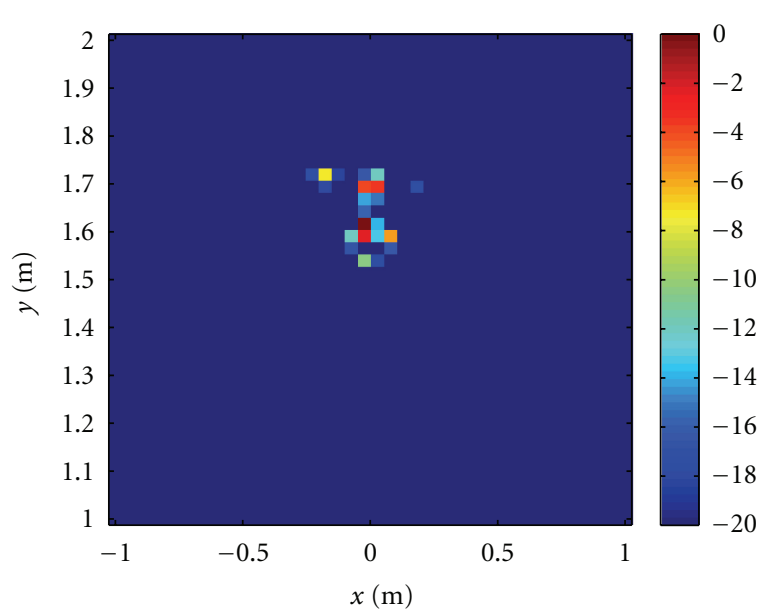

(e)

FIGURE 8: Imaging results of a human behind the wall (a) target scene, (b) conventional pulse radar measurement, (c) TDBP result, (d) CS measurement, and (e) CS result. 
we take 20 inner product measurements at each of the 41 antenna locations and the resulting 820 measurements are shown in Figure 8(d). Figure 8(e) provides the imaging result using the proposed CS imaging algorithm. Comparing Figures 8(c) and 8(e), we see that the CS imaging result accurately locates the human with reduced measurement data and is sparser and less cluttered than the TDBP image. The ISNR for the TDBP and CS imaging results are $22.5 \mathrm{~dB}$ and $41.1 \mathrm{~dB}$, respectively. The sparse constraint optimization seeks a solution to the optimization problem in (14) with as few as possible nozero elements, thus producing more desirable imaging results.

\section{Conclusion}

Conventional UWB impulse through-the-wall radar faces considerable challenges, such as large amount of data measurements, long data acquisition and processing times, and huge data storage requirements. In TWRI, it is desirable to reduce the space-time measurements so as to relax constraints on target motions and ease requirements on imaging system setting and design. In this paper, the impulse radar through-the-wall imaging problem was cast within the framework of compressive sensing. Rather than sampling the time-domain signal at or above the Nyquist rate, the RMPI architecture was employed for the CS projection measurement and led to significant data reduction. Sparsity of the target space was exploited to solve the TWRI problem using sparse constraint optimization. Numerical imaging results of point-like and spatially extended targets clearly showed the merits of applying CS in urban sensing applications.

\section{Acknowledgments}

This work was supported in part by ONR under Grant N00014-11-1-0576 and in part by ARL and ARO under Contract W911NF-11-1-0536.

\section{References}

[1] M. Amin and K. Sarabandi, "Remote sensing of building interior," IEEE Transactions on Geoscience and Remote Sensing, vol. 47, no. 5, Article ID 4812107, pp. 1267-1268, 2009.

[2] M. Amin, Ed., Through-the-Wall Radar Imaging, CRC Press, 2010.

[3] F. Ahmad, M. G. Amin, and S. A. Kassam, "Synthetic aperture beamformer for imaging through a dielectric wall," IEEE Transactions on Aerospace and Electronic Systems, vol. 41, no. 1, pp. 271-283, 2005.

[4] L. P. Song, C. Yu, and Q. H. Liu, "Through-wall imaging (TWI) by radar: 2-D tomographic results and analyses," IEEE Transactions on Geoscience and Remote Sensing, vol. 43, no. 12, pp. 2793-2798, 2005.

[5] W. Zhang, A. Hoorfar, C. Thajudeen, and F. Ahmad, "Full polarimetric beam-forming algorithm for through-the-wall radar imaging," Radio Science, vol. 46, no. 5, 2011.

[6] M. Dehmollaian and K. Sarabandi, "Refocusing through building walls using synthetic aperture radar," IEEE Transactions on Geoscience and Remote Sensing, vol. 46, no. 6, Article ID 4497843, pp. 1589-1599, 2008.
[7] M. G. Amin and F. Ahmad, "Wideband synthetic aperture beamforming for through-the-wall imaging," IEEE Signal Processing Magazine, vol. 25, no. 4, pp. 110-113, 2008.

[8] W. Zhang, A. Hoorfar, and L. Li, "Through-the-wall target localization with time reversal music method," Progress in Electromagnetics Research, vol. 106, pp. 75-89, 2010.

[9] R. Solimene, F. Soldovieri, G. Prisco, and R. Pierri, "Threedimensional through-wall imaging under ambiguous wall parameters," IEEE Transactions on Geoscience and Remote Sensing, vol. 47, no. 5, Article ID 4804799, pp. 1310-1317, 2009.

[10] L. Borcea, G. Papanicolaou, C. Tsogka, and J. Berryman, "Imaging and time reversal in random media," Inverse Problems, vol. 18, no. 5, pp. 1247-1279, 2002.

[11] RANEY RK, "Synthetic aperture imaging radar and moving targets," IEEE Transactions on Aerospace and Electronic Systems, vol. 7, no. 3, pp. 499-505, 1971.

[12] D. L. Donoho, "Compressed sensing," IEEE Transactions on Information Theory, vol. 52, no. 4, pp. 1289-1306, 2006.

[13] E. J. Candes and M. B. Wakin, "An introduction to compressive sampling: a sensing/sampling paradigm that goes against the common knowledge in data acquisition," IEEE Signal Processing Magazine, vol. 25, no. 2, pp. 21-30, 2008.

[14] E. J. Candès, J. Romberg, and T. Tao, "Robust uncertainty principles: exact signal reconstruction from highly incomplete frequency information," IEEE Transactions on Information Theory, vol. 52, no. 2, pp. 489-509, 2006.

[15] J. Romberg, "Imaging via compressive sensing," IEEE Signal Processing Magazine, vol. 25, no. 2, pp. 14-20, 2008.

[16] M. Lustig, D. Donoho, and J. M. Pauly, "Sparse MRI: the application of compressed sensing for rapid MR imaging," Magnetic Resonance in Medicine, vol. 58, no. 6, pp. 1182-1195, 2007.

[17] J. H. G. Ender, "On compressive sensing applied to radar," Signal Processing, vol. 90, no. 5, pp. 1402-1414, 2010.

[18] R. Baraniuk and P. Steeghs, "Compressive radar imaging," in Proceedings of the IEEE 2007 Radar Conference, pp. 128-133, Waltham, Ma, USA, April 2007.

[19] A. C. Gurbuz, J. H. McClellan, and W. R. Scott, "Compressive sensing for subsurface imaging using ground penetrating radar," Signal Processing, vol. 89, no. 10, pp. 1959-1972, 2009.

[20] A. C. Gurbuz, J. H. McClellan, and W. R. Scott, "A compressive sensing data acquisition and imaging method for stepped frequency GPRs," IEEE Transactions on Signal Processing, vol. 57, no. 7, pp. 2640-2650, 2009.

[21] M. A. Herman and T. Strohmer, "High-resolution radar via compressed sensing," IEEE Transactions on Signal Processing, vol. 57, no. 6, pp. 2275-2284, 2009.

[22] Y. S. Yoon and M. G. Amin, in Signal Processing, Sensor Fusion, and Target Recognition XVII, vol. 6968 of Proceedings of SPIE, p. 69681A, Orlando, Fla, USA, March 2008.

[23] L. C. Potter, E. Ertin, J. T. Parker, and M. Çetin, "Sparsity and compressed sensing in radar imaging," Proceedings of the IEEE, vol. 98, no. 6, Article ID 5420035, pp. 1006-1020, 2010.

[24] Q. Huang, L. Qu, B. Wu, and G. Fang, "UWB through-wall imaging based on compressive sensing," IEEE Transactions Geoscience and Remote Sensing, vol. 48, no. 3, pp. 1408-1415, 2010.

[25] Y. S. Yoon and M. G. Amin, "Imaging of behind the wall targets using wideband beamforming with compressive sensing," in Proceedings of the IEEE/SP 15th Workshop on Statistical Signal Processing, (SSP '09), pp. 93-96, Cardiff, UK, September 2009.

[26] Y. S. Yoon and M. G. Amin, "Through-the-wall radar imaging using compressive sensing along temporal frequency 
domain," in Proceedings of the IEEE International Conference on Acoustics, Speech, and Signal Processing, (ICASSP'10), pp. 2806-2809, Dallas, Tex, USa, March 2010.

[27] M. G. Amin, F. Ahmad, and W. Zhang, "A compressive sensing approach to moving target indication for urban sensing," in Proceedings of the IEEE Radar Conference (RadarCon'11), Kansas City, Mo, USA, May 2011.

[28] M. Leigsnering, C. Debes, and A.M. Zoubir, "Compressive sensing in through-the-wall radar imaging," in Proceedings of the IEEE Int. Conf. Acoustics, Speech, and Signal Process, pp. 4008-4011, Prague, Czech Republic, 2011.

[29] E. L. Targarona, M. Amin, F. Ahmad, and M. Nájar, "Wall mitigation techniques for indoor sensing within the CS framework," in Proceedings of the 7th IEEE Workshop Sensor Array and Multi-Channel Signal Process, Hoboken, NJ, USA, June 2012.

[30] E. L. Targarona, M. Amin, F. Ahmad, and M. Nájar, "Compressive sensing for through wall radar imaging of stationary scenes using arbitrary data measurements," in Proceedings of the 11th International Conference on Information Sciences, Signal Processing and their Applications (ISSPA'12), Montreal, Canada, July 2012.

[31] G. Wang and M. G. Amin, "Imaging through unknown walls using different standoff distances," IEEE Transactions on Signal Processing, vol. 54, no. 10, pp. 4015-4025, 2006.

[32] Y. S. Yoon and M. G. Amin, "Spatial filtering for wallclutter mitigation in through-the-wall radar imaging," IEEE Transactions on Geoscience and Remote Sensing, vol. 47, no. 9, Article ID 5109740, pp. 3192-3208, 2009.

[33] F. H. C. Tivive, A. Bouzerdoum, and M. G. Amin, "An SVDbased approach for mitigating wall reflections in throughthe-wall radar imaging," in Proceedings of the IEEE Radar Conference, pp. 519-524, Kansas City, Mo, USA, 2011.

[34] C. Thajudeen, A. Hoorfar, and W. Zhang, "Estimation of frequency-dependent parameters of unknown walls for enhanced through-the-wall imaging," in Proceedings of the IEEE International Symposium Antennas and Propagation (APSURSI'11), pp. 3070-3073, Spokane, Wash, USA, 2011.

[35] S. S. Chen, D. L. Donoho, and M. A. Saunders, "Atomic decomposition by basis pursuit," SIAM Journal on Scientific Computing, vol. 20, no. 1, pp. 33-61, 1998.

[36] E. Candes and J. Romberg, "L1-magic : recovery of sparse signals via convex programming," http://users. ece.gatech.edu/ justin/l1magic/downloads/l1magic.pdf.

[37] P. Boufounos, M. F. Duarte, and R. G. Baraniuk, "Sparse signal reconstruction from noisy compressive measurements using cross validation," in Proceedings of the /SP 14th WorkShoP on Statistical Signal Processing, (SSP'07), pp. 299-303, Madison, Wis, USA, August 2007.

[38] W. J. Zheng, Z. Q. Zhao, Z. P. Nie, and Q. H. Liu, "Evaluation of trm in the complex through wall environment," Progress in Electromagnetics Research, vol. 90, pp. 235-254, 2009. 

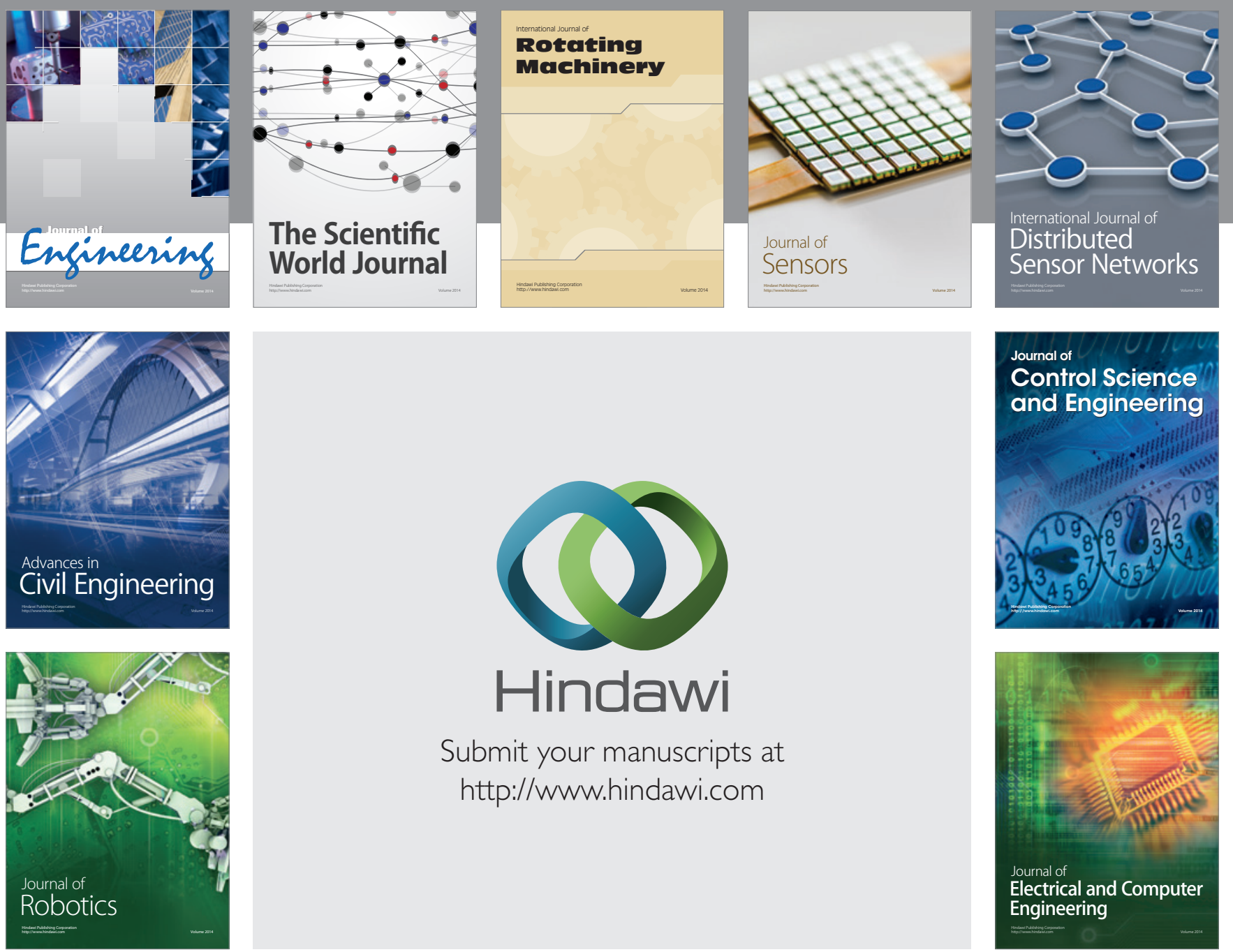

Submit your manuscripts at

http://www.hindawi.com
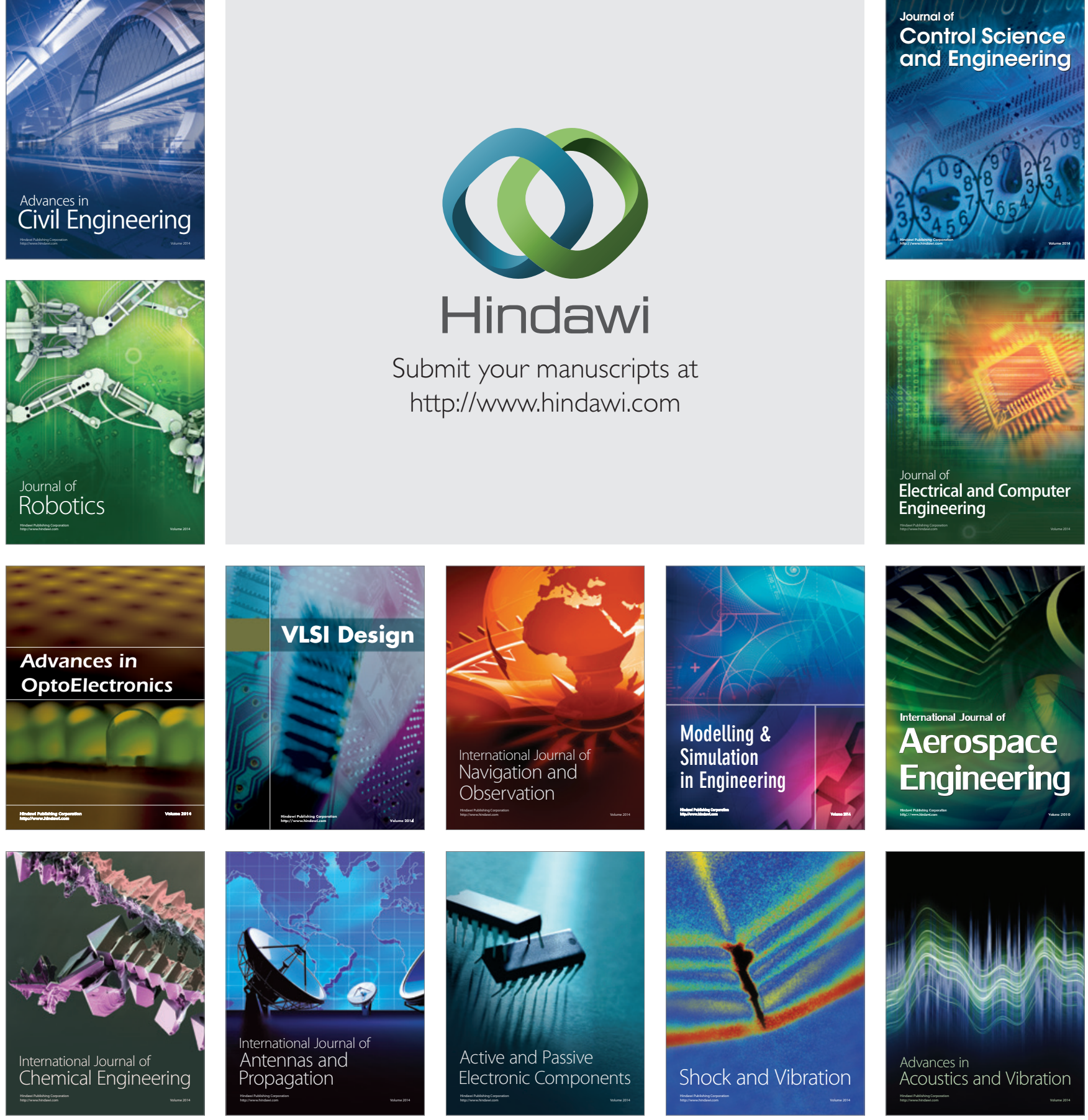